healthcare assistants (HCA) to perform LSMs. The aim of this review was to assess the impact of this change on the quality of LSM as measured by success rate and failed scans.

Methods A transient elastography service delivered by trained specialist liver nurses was set up in our hospital in May 2010. In July 2013, 3 HCAs were trained to carry out LSM using a Fibroscan ${ }^{\circledR}$. The HCAs were initially trained by the manufacturers of the Fibroscan ${ }^{\circledR}$ unit (Echosens Europe) and then underwent a period of formally observed training with formative and summative work place based assessments. After competency was ascertained, the HCAs were independently allowed to carry out LSMs. A retrospective review of all LSM reports from January 2013 to December 2013 was carried out and success rate of the tests were recorded. Any repeat requests due to failure were also recorded.

Results A total of 876 LSM were performed during the review period. 542 LSMs were performed by trained nurses and 334 by trained HCAs. There was no statistically significant difference in the mean success rate between nurses (96\% SD 11.9\%) and HCAs $(96.4 \%$ SD 11.7\%) ( $\mathrm{p}=0.699,2$ sample T Test) nor the proportion of LSMs with $100 \%$ success rates between the two groups (78.4 vs. $82.3 \% \mathrm{p}=0.151$, Fisher's exact test). Furthermore, there were no statistical differences in any central measure of the observed interquartile ranges of the reported LSM between the 2 groups $(p=0.255)$. No LSM was repeated when performed by HCA for reasons of failure.

Conclusion LSM using a Fibroscan ${ }^{\circledR}$ can be accurately performed by appropriately trained HCAs. The introduction of this change in practice has allowed a reduction in waiting time for LSM to within 2 weeks without affecting the quality of the service and allowed a more efficient use of resources. A high quality transient elastography service can be delivered by HCAs.

Disclosure of Interest None Declared.

\section{PTH-042 CLARIFYING THE REFERRAL PATHWAYS FOR PATIENTS DIAGNOSED WITH GASTROINTESTINAL CANCER: IS THE 'RED-FLAG' PATHWAY WORKING?}

JK Eccles*, PM Lynch. Gastroenterology, Antrim Area Hospital, Antrim, UK

\subsection{6/gutjnl-2014-307263.488}

Introduction The Cancer Access or 'Red-Flag' referral pathway was introduced to facilitate appropriate referral between primary and secondary care for cases of suspected cancer. Despite this, many cases of upper and lower gastrointestinal (GI) cancer are diagnosed through 'routine' referral grading, emergency presentations, and in the case of colorectal cancers, the bowel cancer screening (BCS) programme. The purpose of this study was to assess the original referral pathway for patients diagnosed with gastrointestinal cancer within our Health Trust and the effects, if any, on patient outcomes.

Methods We looked at a random sample of clinical notes of 107 patients diagnosed with a GI cancer between April 2011 and December 2012 within our trust (56 patients with lower and 51 patients with upper GI cancer) to determine referral source and grade, whether red-flag criteria were positive, staging and outcomes, whether curative or palliative.

Results 58 patients (54\%) diagnosed with upper or lower GI cancer had been referred to the Trust via GP (with 22\% seen initially at clinic and $32 \%$ at direct access endoscopy), 5 patients (5\%) had been referred to clinic by another physician, 28 patients $(26 \%)$ attended through casualty, 10 patients were diagnosed through the BCS programme (9\%), 5 oesophageal cancers (5\%) through
Barrett's surveillance, and 1 colorectal cancer (1\%) through polyp surveillance. All 27 lower GI cancer patients initially referred by their GP had 'red-flag' symptoms, but only 12 (44\%) were referred with an initial 'red-flag' grade; similarly, all 31 upper GI cancer patients initially referred by GP had 'red-flag' symptoms, but only 15 patients (48\%) were initially referred as 'red-flag'. Of the 51 upper GI cancer patients, 20 underwent curative treatment; 11 such patients were referred from GP (5 of which were originally referred as 'red-flag'), 3 from another physician, 5 from Barrett's surveillance and 1 casualty self-presenter. Of the 56 lower GI cancer patients, 33 underwent curative treatment -17 referred from GP (only 7 originally referred as 'red-flag'), 6 casualty selfpresenters, and all $10 \mathrm{BCS}$ patients (all Dukes' A-B). Of the 54 palliative cases of either upper or lower GI cancer, only 15 of the 30 patients referred by their GP were referred through the 'red-flag' pathway.

Conclusion All patients diagnosed with a GI cancer that were originally referred from primary care had evidence to satisfy 'red-flag' referral, although less than half of these were referred through the 'red-flag' pathway. This study highlights the need for ongoing education and reinforcement of the 'red-flag' referral criteria.

Disclosure of Interest None Declared.

\section{PTH-043 OUR EXPERIENCE OF A PHARMACIST LED IMMUNOMODULATOR (IMD) CLINIC: A NOVEL SERVICE IN A DISTRICT GENERAL HOSPITAL}

V Sathyanarayana, K Kapur*, F Fuertes, E Said, A Soliman, D West, G Smith, N Sanassee, S Riyaz. Gastroenterology, Barnsley District General Hospital, Barnsley, UK

\subsection{6/gutjnl-2014-307263.489}

Introduction Increasing numbers of patients are being treated with immunomodulators (IMD) for inflammatory bowel disease (IBD) and autoimmune hepatitis (AIH). This needs intensive monitoring and impacts by increasing clinic waiting times. After approval from the Quality and Safety Board of the Trust, a pharmacist led IMD clinic was established in 2012 to manage patients initiated on IMD for initial monitoring and dose titration with a view to reduced clinic visits.

Methods Patients were referred to the pharmacist led clinic by the gastroenterologists and IBD nurse specialist for commencing and monitoring of IMD after initial counselling. Screening blood tests including the TPMT assay were checked prior to commencing the IMD as per agreed protocol. The pharmacist issued prescriptions and patients were given blood forms for weekly tests for the initial two months, fortnightly for the next two months and three monthly thereafter. Results were monitored by the pharmacist and patients were offered a choice of telephone or email consultations with the pharmacist for subsequent appointments. The pharmacist had easy access to advice from the clinician in the event of adverse effects. After initial stabilisation patients were referred back to the GP or the referring clinician for follow up.

Results 81 patients were referred to the pharmacist led IMD clinic between October 2012-2013 [(50F); Median age 44 (range 1976)]. Indications for treatment were IBD $(n=73)$ [ulcerative colitis $(n=33)$, Crohn's $(n=40)]$ and AIH $(n=8)$. Twenty seven patients $(33.3 \%)$ experienced side effects between weeks 2 to 6 of initiation of treatment. These were nausea or vomiting $16 \%(\mathrm{n}=$ 13), skin rash $1 \%(n=1)$, fatigue $1 \%(n=1)$, myalgia $1 \%(n=1)$, intolerance $1 \%(\mathrm{n}=1)$ and stomach cramps $1 \%(\mathrm{n}=1)$. Abnormal blood tests were noted in 23.4\% $(\mathrm{n}=19)$ patients. These 